\title{
MulTi-VieW FACE DeTECTION BASED ON KERNEL Principal COMPONENT ANALYSIS AND KERNEL SUPPORT VECTOR TECHNIQUES
}

\author{
Muzhir Shaban Al-Ani ${ }^{1}$ and Alaa Sulaiman Al-Waisy ${ }^{2}$ \\ Department of Computer Science, College of Computer, Al-Anbar University, Iraq. \\ ${ }^{1}$ muzhir_shaban@yahoo.com_ ${ }^{2}$ king_alaa87@yahoo.com
}

\begin{abstract}
Detecting faces across multiple views is more challenging than in a frontal view. To address this problem, an efficient approach is presented in this paper using a kernel machine based approach for learning such nonlinear mappings to provide effective view-based representation for multi-view face detection. In this paper Kernel Principal Component Analysis (KPCA) is used to project data into the view-subspaces then computed as view-based features. Multi-view face detection is performed by classifying each input image into face or non-face class, by using a two class Kernel Support Vector Classifier (KSVC). Experimental results demonstrate successful face detection over a wide range of facial variation in color, illumination conditions, position, scale, orientation, $3 D$ pose, and expression in images from several photo collections.
\end{abstract}

\section{KEYWORDS}

Face Detection, Face Recognition, Kernel Principal Component Analysis, Kernel Support Vector Machine.

\section{INTRODUCTION}

Face detection is the first stage of an automated face recognition system, since a face has to be located in the overall image before it is recognized[1].As computers become faster and more affordable, many applications that use face detection/ localization are becoming an integral part of daily life. For example, face identification system, face tracking, video surveillance and security control system, and human computer interface. Those applications often require detected and segmented human face which is ready to be processed[2],[3]. However detecting a face under various environments is still challenging work. Some factors make face detection difficult. One is the variety of colored lighting sources; another is that facial features such as eyes may be partially or wholly occluded by a shadow generated by a bias lighting direction; and others are race and different face poses with/without glasses. Finally because faces are not rigid and have a high degree of variability in size, shape, color, and texture[4]. Therefore detection rate and the number of false positives are important factors in evaluating face detection systems[5]. This paper describes progress toward a system which can detect faces regardless of pose reliably and in realtime. In the presented system a kernel machine learning based approach for extracting nonlinear features of face images and using them for multi-view face detection. KPCA is applied on a set of view-labeled face images to learn nonlinear view- subspaces. Nonlinear features are the

DOI : 10.5121/ijsc.2011.2201 
projections of the data onto these nonlinear view-subspaces. Face detection is performed by using $\mathrm{KSVC}$ as the classifying function, based on the nonlinear features. One distinctive advantage this type of classifiers has over traditional neural networks is that Support Vector Machines (SVM) achieve better generalization performance. While neural networks such as Multiple Layer Perceptrons (MLPs) can produce low error rate on training data, there is no guarantee that this will translate into good performance on test data[2]. The literature on SVMs includes many types of pattern recognition topics like face authentication, face recognition, object detection, text classification, image classification and voice identification[6]. The results show that the proposed approach yields high detection and low false alarm rates in face detection, even under different conditions of lighting or when add some noise to the testing images. The remainder of the paper is organized as follows: Section 2 introduces basic concepts of kernel learning methods that is, KSVC and KPCA .Section 3 introduces the literature survey about the face detection system. Section 4 the implemented face detection system. Section 5 shows experimental results. Section 6 the conclusion.

\section{FACE DETECTION}

Face detection is a two-class classification problem, which is used to discriminate face patterns from background. Many factors, as explain above contribute to difficulties in face detection. Face patterns themselves include many elements that vary greatly with different persons and environment, such as face pose, skin color, face components and facial expression. The multiple poses make the assumption of convexity in feature space invalid. Furthermore, the unpredicted noise and illumination condition in the complex scene cause a lot of false detections[7]. To address this problems, an efficient approach is presented in this paper. The kernel methods generalize linear SVC and PCA to nonlinear ones. The trick of kernel methods is to perform dot products in the feature space by using kernel functions in input space so that the nonlinear mapping is performed implicitly in the input space.

\subsection{Kernel Principal Components Analysis (KPCA)}

The KPCA is the nonlinear version of PCA that is constructed by using a specified kernel function. As a simple description for the PCA. The PCA is used to lower the dimensional space of the feature to reduce the time complexities [8]. Eigenvectors of the covariance matrix of the face images constitute the eigenfaces. The dimensionality of the face feature space is reduced by selecting only the eigenvectors possessing largest eigenvalues Once the new face space is constructed, when a test image arrives, it is projected onto this face space to yield the feature vector the representation coefficients in the constructed face space. The classifier decides for the identity of the individual. according to a similarity score between the test image's feature vector and the PCA feature vectors of the individuals in the database[9].Using PCA for eigenfaces method, feature vectors identifying each image can be obtained as follows:

Given a set of examples in $R^{N}$ represented by column vectors, subtract them by the their mean vector to obtain the centered examples $\mathrm{x}_{\mathrm{i}} \in R^{N}(\mathrm{i}=1, \ldots, \mathrm{m})$. The covariance matrix is

$$
\mathrm{C}=\frac{1}{\mathrm{~m}} \sum_{\mathrm{j}=1}^{\mathrm{m}} \mathrm{x}_{\mathrm{j}} \mathrm{x}_{\mathrm{j}}^{\mathrm{T}} \ldots \ldots \ldots \ldots \ldots \ldots \ldots
$$

Linear PCA is an algorithm which diagonalizes the covariance matrix by performing a linear transformation. By using a nonlinear mapping, the data set can be mapped into a higher dimensional feature space $H$. The representation of features in this high dimensional feature space 
helps the classifier to perform better. Fortunately, for certain feature spaces $H$ there is a function for computing scalar products in feature spaces. This is known as a kernel function. By using a kernel function, every linear algorithm that uses scalar products can be implicitly executed in $H$ without explicitly knowing the mapping $\Phi$, constructing a nonlinear version of a linear algorithm. The nonlinear version of PCA that is constructed by using a kernel function is known as kernel principal component analysis KPCA. Let us now generalize classic PCA to kernel PCA. Let $\Phi: x \in R^{N} \rightarrow X \in H$ be a mapping from the input space to a high dimensional feature space[10]. The covariance matrix in $H$ is

$$
\overline{\mathrm{C}}=\frac{1}{\mathrm{~m}} \sum_{\mathrm{j}=1}^{\mathrm{m}} \Phi\left(\mathrm{x}_{\mathrm{j}}\right) .\left(\mathrm{x}_{\mathrm{j}}\right)^{\mathrm{T}} \ldots \ldots \ldots \ldots \ldots \ldots
$$

To do this, we have to find the eigenvalues $\lambda \geq 0$ and eigenvectors satisfying

$$
\lambda v=\bar{C} v
$$

All solutions $v$ with $\lambda \neq 0$ must lie in the span of $\Phi\left(\mathrm{x}_{1}\right), \Phi\left(\mathrm{x}_{2}\right), \ldots, \Phi\left(\mathrm{x}_{\mathrm{m}}\right)$. Hence Eq. 2 is equivalent to

$$
\lambda\left(\Phi\left(x_{k}\right) \cdot v\right)=\left(\Phi\left(x_{k}\right) \cdot \bar{C} v\right)
$$

For $k=1,2, \ldots . ., m$

Because all $v$ for nonzero $\lambda$ must lie in the span of the $\boldsymbol{x}_{k}$ 's, there exist coefficients $\alpha_{i}$ such that

$$
v=\sum_{i=1}^{m} \alpha_{i} \Phi\left(x_{i}\right) \ldots \ldots \ldots \ldots \ldots \ldots \text { (5) }
$$

Defining the matrix $K=\left[K_{\mathrm{i}, \mathrm{j}}\right]_{\mathrm{m} \times \mathrm{m}}$, the eigenvalue problem can be converted into the following:

$$
m \lambda \alpha=K \alpha
$$

for nonzero eigenvalues. Sort $\lambda_{i}$ in descending order and use the first $\mathrm{M}<=\mathrm{m}$ principal components $v_{\mathrm{i}}$ as the basis vector in $\mathrm{H}$ (In fact ,there are usually some zero eigenvalues, in which case $\mathrm{M}<\mathrm{m}$ ). The $\mathrm{M}$ vectors spans a linear subspace, called KPCA subspace, in $\mathrm{H}$. The projection of a point $\mathrm{x}$ onto the $\mathrm{k}$-th kernel principal component $v_{\mathrm{k}}$ is calculated as:

$$
\begin{gathered}
\left(v_{k} \cdot \Phi(x)\right)=\sum_{i=1}^{m} a_{k, i}\left(\Phi\left(x_{i}\right) \cdot \Phi(x)\right) \\
=\sum_{i=1}^{m} a_{k, i} K\left(x_{i}, x\right) \ldots \ldots \ldots \ldots(7)
\end{gathered}
$$




\subsection{Kernel Support Vector Machines (KSVMs)}

Support vector machines (SVMs) are a popular method for binary classification. SVMs can be seen as an extension of the perceptron, which tries to find a hyperplane that separates the data [11]. A more detailed discussion of the theory and applications of SVMs can be found in [6].Consider the problem of separating the set of training vectors belonging to two classes, given a set of training data $\left(\mathrm{x}_{\mathrm{i}}, \mathrm{y}_{\mathrm{i}}\right), \ldots \ldots,\left(x_{m}, y_{m}\right)$ where $\mathrm{x}_{\mathrm{i}} \in \mathrm{R}^{\mathrm{N}}$ is a feature vector and $y_{i} \in\{-1,+1\}$ its class label. If the two classes are linearly separable, there exists a separating hyperplane $(w, b)$ is given by the function:

$$
f(x)=\operatorname{sign}\left(\sum_{i=1}^{m} \alpha_{i} y_{i}\left(x_{i} \cdot x\right)+b\right) \ldots \ldots .
$$

In real-life problems it is rarely the case that positive and negative samples are linearly separable. Non-linear support vector classifiers map input space $\mathrm{R}^{\mathrm{N}}$ to a high dimensional feature space $H$ by $x \rightarrow \Phi(x) \in H$ such that the mapped data is linearly separable in the feature space [12]. In short, a hard margin SVM solves the quadratic program (QP1) which is given as follows. Find the Lagrange multipliers $\{\alpha\}_{i=1}^{N}$ that maximize the objective function:

$$
Q(\alpha)=\sum_{i=1}^{m} \alpha_{i}-\frac{1}{2} \sum_{i=1}^{m} \sum_{j=1}^{m} \alpha_{i} \alpha_{j} y_{i} y_{j} k\left(x_{i}, x_{j}\right) \ldots
$$

Subject to

$$
\sum_{i=1}^{m} \alpha_{i} y_{j}=0, \quad 0 \leq \alpha_{i} \leq C
$$

Where $\mathrm{C}$ is a user-specified positive parameter. If $0 \leq \alpha_{\mathrm{i}} \leq \mathrm{C}$, the corresponding data points are called support vectors. Having the Lagrange multipliers, the optimum weight vector $\mathbf{w}$ could be computed by:

$$
w=\sum_{i=1}^{m} \alpha_{i} y_{i} \Phi\left(x_{i}\right) \ldots \ldots \ldots \ldots \ldots \ldots
$$

By taking the samples with $0<\alpha_{i}<C$, the bias could be calculated by

$$
b=\frac{1}{N o . S V} \sum_{x_{i} \in S V}\left(\frac{1}{y_{i}}-\sum_{x_{j} \in S V} \alpha_{i} y_{i} K\left(x_{j}, x_{i}\right)\right) \ldots \ldots \ldots
$$


Where No.SV is the number of support vectors with, $0<\alpha_{i}<C$. Next, a separating hyper plane is computed in $\mathrm{H}$. The decision function becomes

$$
f(x)=\operatorname{sign}\left(\sum_{i=1}^{N s} \alpha_{i} y_{i} K\left(x_{i}, x\right)+b\right) \ldots \ldots \ldots \ldots
$$

Where Ns is the number of support vectors and $\mathrm{K}($, ) is a kernel function. Several kernels are possible including radial basis functions, polynomial and sigmoid kernels. The choice of the kernel and kernel parameters(e.g. the degree of the polynomial kernel) has to be made by the user, and the optimal choices are problem dependent [13],[14].

\section{LITERATURE SURVEY}

Face detection has been a typical active research domain for decades because it can be applied in many fields as concluded in [15]. In recent years, face detection algorithm access a significant development, but in comparison with the human visual system is still a big gap because it has lots of variations of image appearance, such as pose variation (front, non-front), occlusion, image orientation, illuminating condition and facial expression. A lot of research is going on in the area of human face detection at present. Many researchers have proposed different approaches to address face detection problem such as:

Paola Campadelli, Raffaella Lanzarotti proposed a face detection algorithm the key idea being to determine roughly the skin regions of a 2D color image and searching for eyes through them. The technic is based on a Support Vector Machine trained to separate sub images representing eyes from others. The algorithm can be used in face image database management systems both as a first step of a person identification, and to discriminate the images on the basis of the number of faces in them [16]. Lamiaa Mostafa and Sherif Abdelazeem combined two algorithms for face detection to achieve better detection rates. The two algorithms are skin detection and neural networks. In the first module of the system a skin color model based on normalized RGB color space is built and used to detect skin regions. The detected skin regions are the face candidate regions. In the second module of the system, the neural network is created and trained with training set of faces and non-faces. The network used is a two layer feed-forward network but this work can be achieves better results only when the faces in the image having upright frontal faces [17]. Wang Chuan-xu and Li Xue proposed a face detection method employing BP network combined with Gabor wavelet transform. Sample Features are represented by Gabor description for BP network training. In this work the characteristics of Gabor wavelet transformation are exploited such as the spatial locality, frequency selectivity and direction selectivity etc, Gabor features of the frequency and direction information are extracted from input image, which reflect face local area peculiarity, and let the extracted attributes be the input to the BP nerve network. This processing method is the exact simulation of human being's thinking mode, that is, when a brain identifies the face, only the eminent traits derived from a human face are valuable to human brain for recognition [18]. Jizeng Wang and Hongmei Yang proposed a hierarchical face detection method by using the template matching algorithm and 2DPCA algorithm. The method includes two different classifiers. The first one is called rough classifier which filtrates the most of the non-face .The second one is a core classifier, which uses 2DPCA algorithm to detect the face based on the result from the first classifier [19]. Min-Quan Jing and Ling-Hwei proposed a 
method to detect a face with different poses under various environments. On the basis of skin color information, skin regions are first extracted from an input image. Next, the shoulder part is

cut out by using shape information and the head part is then identified as a face candidate. For a face candidate, a set of geometric features is applied to determine if it is a profile face. If not, then a set of eyelike rectangles extracted from the face candidate and the lighting distribution are used to determine if the face candidate is a nonprofile face [20]. Yongqiu Tu, Faling Vi and et al proposed a face detector has been designed using multi-classifier combination method. The proposed detector composes of three classifiers: Skin color detector, AdaBoost detector based on haar-like features, and eye-mouth detector, a semi-serial architecture is designed to combine the three detectors, which set up the division and cooperation system and draw on each other's merits to implement the quick and efficient facial detection [21]. Payman Moallem and Bibi Somayeh proposed a fuzzy rule based system for pose, size and position independent face detection in color image. Subtractive clustering method is also applied to decide on the numbers of membership functions. In the proposed system, skin-color, lips position, face shape information and ear texture properties are the key parameters fed to the fuzzy rule based classifier to extract face candidate in an image. Furthermore, the applied threshold on the face candidates is optimized by genetic algorithm [22].

\section{IMPLEMENTED SYSTEM DESIGN}

The proposed system can be summarized in the four steps as shown in the figure (1). Image size normalization, Median filtering, Feature extraction algorithm by using the KPCA and Classification algorithm based on the KSVC.

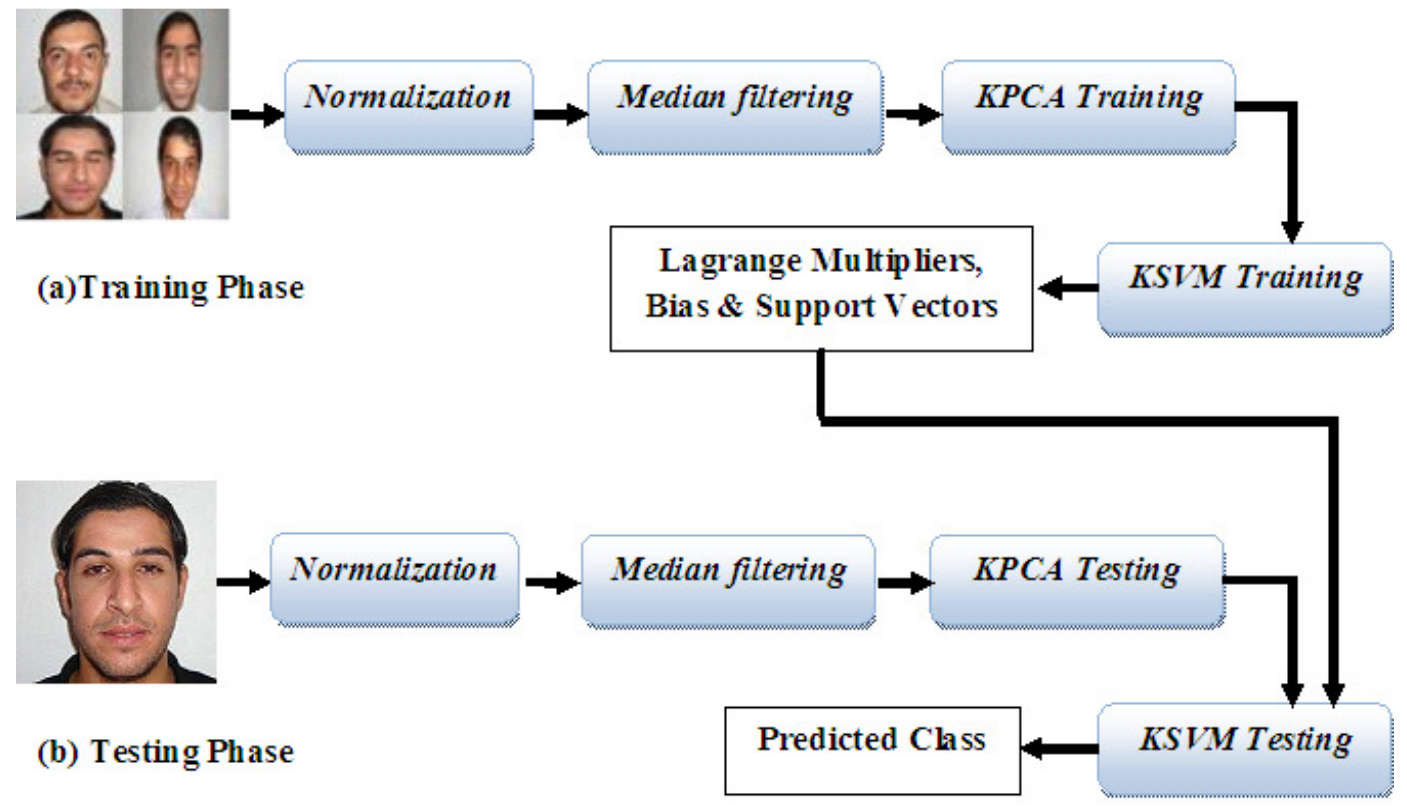

Figure 1. The block diagram of the proposed face detection system. 


\subsection{Image size normalization}

Size normalization is an important pre-processing technique in face detection and recognition. Although various effective learning-based methods have been proposed. It is usually done to change the acquired image size to a default image size. In this paper the default image size is 256 $\mathrm{x} 256$, on which the proposed face detection system operates.

\subsection{Median filtering}

Median filtering follows this basic prescription. The median filter is normally used to reduce noise in an image especially obtained from a camera, somewhat like the mean filter. However, it often does a better job than the mean filter of preserving useful detail in the image. This class of filter belongs to the class of edge preserving smoothing filters which are non-linear filters. This means that for two images $\mathrm{A}(\mathrm{x})$ and $\mathrm{B}(\mathrm{x})$ :

$$
\operatorname{median}[A(x)+B(x)] \neq \operatorname{median}[A(x)]+\operatorname{median}[B(x)] \ldots \ldots \ldots(14)
$$

These filters smooth the data while keeping the small and sharp details. The median is just the middle value of all the values of the pixels in the neighborhood. Note that this is not the same as the average (or mean); instead, the median has half the values in the neighborhood larger and half smaller. The median is a stronger "central indicator" than the average. In particular, the median is hardly affected by a small number of discrepant values among the pixels in the neighborhood. Consequently, median filtering is very effective at removing various kinds of noise. Figure(2) illustrates an example of median filtering.

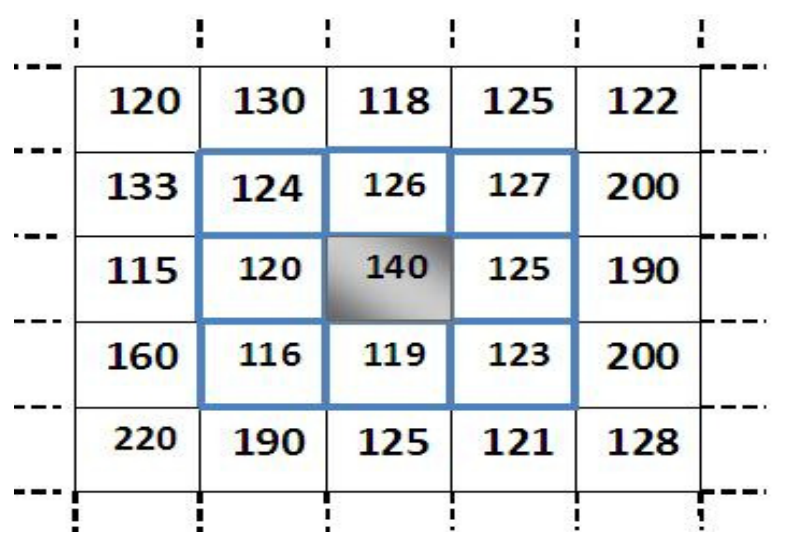

Neighbourhood values:

$116,119,120,123,124$

$125,126,127,140$

Median value: 124

Figure 2. Median filtering operation.

\subsection{Feature Extraction Algorithm}

This algorithm can be achieved based on ability of the KPCA. KPCA feature extraction effectively acts a nonlinear mapping from the input space to an implicit high dimensional feature space. It is hoped that the distribution of the mapped data in the implicit feature space has a simple distribution so that a simple classifier (which need not to be a linear one) in the high 
dimensional space could work well. The steps to compute the principal components can be summarized as:

- Compute the matrix $K$, see Eq.(2). In this paper the polynomial kernel is used as a kernel function:

$$
K\left(x_{i}, x_{j}\right)=\left(a\left(x_{i} \cdot x_{j}\right)+b\right)^{n} \ldots \ldots \ldots \ldots(15)
$$

Where $\mathrm{a}=0.001 ; \mathrm{b}=-1 ; \mathrm{n}=3$ and $\mathrm{x}_{\mathrm{k}} \in \mathrm{R}^{\mathrm{N}}$ are taken from the face images by rearranging the pixel value order as shown in figure(3).

- To acquire the eigenfaces, the face image data are converted from matrices to vectors, where the vector version of each face is a column in a matrix. For example, for a training set of 250 images each have $256 \times 256$ pixels will be converted into a matrix of that is $65536 \times 250$. This matrix of face vectors uses KPCA to compute the eigenfaces.

- The resulting eigenfaces are then point multiplied with the training set images to filter out outlier data and focus the training on the principal features of the face. The resulting images have their intensities scaled.

- The first $\mathrm{M}=50$ most significant principal components are used as the basic vectors. Which is aiming to train the KSVC to differentiate between face and non-face patterns for face detection.

- Compute projections of a test point onto the eigenvectors.
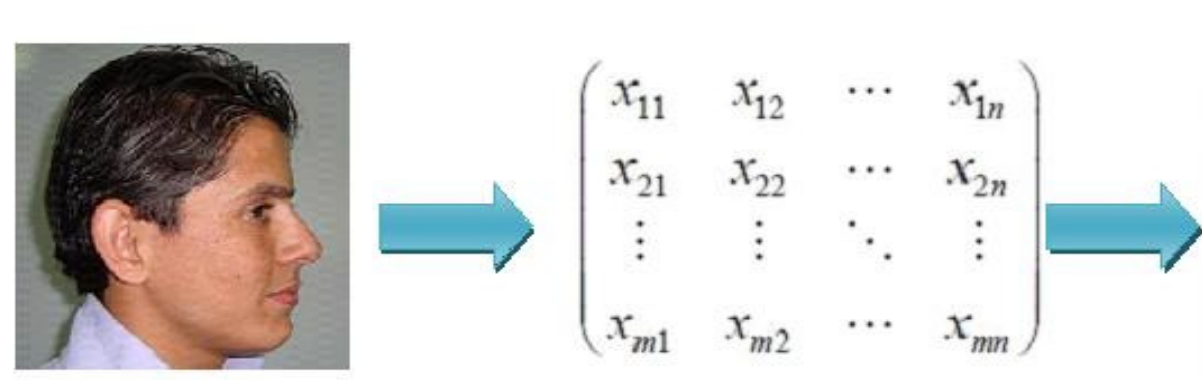

$$
\left(\begin{array}{c}
x_{11} \\
x_{12} \\
\vdots \\
x_{1 n} \\
x_{21} \\
\vdots \\
x_{m n}
\end{array}\right)
$$

Figure 3. Pre-processing step.

\subsection{Classification Algorithm}

SVMs implement complex decision rules by using a non-linear function $\Phi$ to map training points to a high dimensional feature space where the labelled points are separable .A separating hyperplane is founded which maximizes the distance between itself and the nearest training points this distance is called the margin .The hyperplane is, in fact ,represented as a linear combination of the training points . The steps to find an optimal separable hyperplane (decision function ) can be summarized as: 
- After further refining the images using a principal components approach, The resulting processed images are converted into input vectors $x_{i}$ and class values $y_{i}$ where $i \in\{1, \ldots, p$, $\mathrm{p}+1, \ldots, \mathrm{q}\}$ with $\mathrm{p}$ and $\mathrm{q}$ being the number of user and imposter images, respectively, and $\mathrm{x}$ has $\mathrm{M}$ dimensions where the dimension is the number of pixels in the image. For the user images, $y_{\mathbf{j}}=+1$ where $j \in\{1, \ldots, p\}$, and for the imposter images, $y_{\mathbf{k}}=-1$ where $k \in\{p+1$, $\ldots, \mathrm{q}\}$.

- The data are passed to the SVM training along with the kernel type and kernel parameters. In this paper is Radial Basis Function (RBF) Kernel which can be calculated as follow:

$$
K\left(x_{i}, x_{j}\right)=\exp \left(-\operatorname{Sigma} *\left\|x_{i}-x_{j}\right\|^{\wedge} 2\right) \ldots \ldots(1
$$

Where Sigma is the width which is specified by the user.

- The decision function will then be tuned to find the optimal SVM parameters for data. After the training function is complete a training model is returned which will be used for the classification.

- In the testing phase the acquisition and processing steps of the tuning images are the same as that required for the training data except the images in this set are used for classification not training.

- By using the tuning data sets into the SVM classification function the effectiveness of the system and its kernels can be tested. The effectiveness is gauged by overall detection rate and false positive number. Based on the rate for all the test sets the kernel and kernel parameters are adjusted until the kernel and parameter combination with the highest accuracy can be founded.

\section{EXPERIMENTAL RESULTS}

The experiment was done by constructing multi-view face database. This database currently contains colored face images of 50 persons. Each person is photographed against a uniform white background using a single camera and identical settings. For each person, we take 10 photographs. Each photograph has a different combination of viewpoint such as (frontal $0^{\circ}$, right $45^{\circ}$, right $90^{\circ}$, left $45^{\circ}$ and left $90^{\circ}$ ) and facial expression such as (smiling, laughing, neutral and closed eyes). Figure (4) shows the image variations for three persons. 


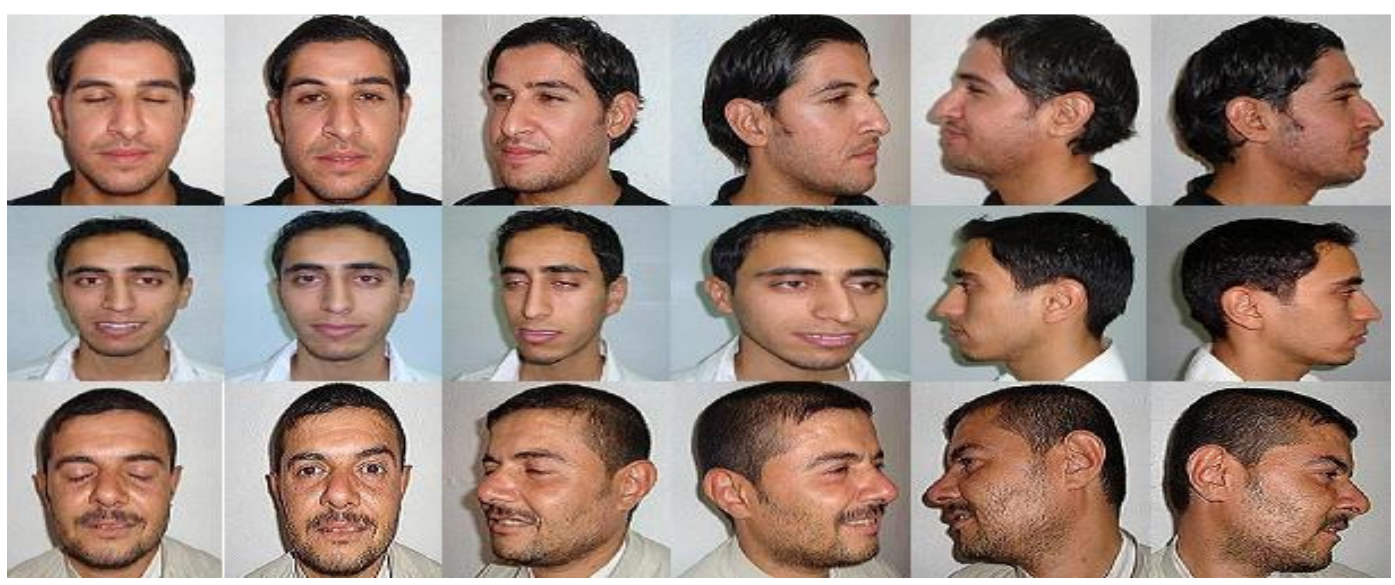

Figure 4. Image variation of some persons in the constructed multi-view face database

In this paper we compare the performance of the implemented system by computing the face detection rate and the number of false negative in five cases face detection. Considering the number of the training and testing images to be 5. In the first case the face images are tested as they are captured. In the second and third cases the illumination conditions of the training and testing images are changed respectively, as shown in the figure (5). In fourth and fifth cases some

ratio of noise is added to the training and testing images respectively (Salt and Pepper type is used in these two cases). The face detection rate and the number of false negative of the proposed system in these five cases are summarized in Table 1.The training time and testing time are summarized in Table 2. Every algorithm used 255 images in the training phase (face and nonface) and the testing time is also calculated for each image.

Table 1. The face detection rate and the number of false negative.

\begin{tabular}{|l|c|c|c|}
\hline The Case Number & Number of Image & False Negative & Detection \\
\hline First Case & 255 & 14 & 94.4 \\
\hline Second Case & 255 & 6 & 97.6 \\
\hline Case Third & 255 & 15 & 94 \\
\hline Fourth Case & 255 & 10 & 96 \\
\hline Fifth Case & 255 & 17 & 93.2 \\
\hline
\end{tabular}

Table 2. The training time and testing time.

\begin{tabular}{|l|c|c|}
\hline \multicolumn{1}{|c|}{ The Method } & Training Time (s) & Testing Time (s) \\
\hline KPCA & 184.522940 & $\mathbf{0 . 1 0 1 2 5 6}$ \\
\hline KSVM & 1.987141 & $\mathbf{0 . 0 0 0 4 2}$ \\
\hline KPCA-KSVM & 186.10081 & $\mathbf{0 . 1 0 1 6 7 6}$ \\
\hline
\end{tabular}




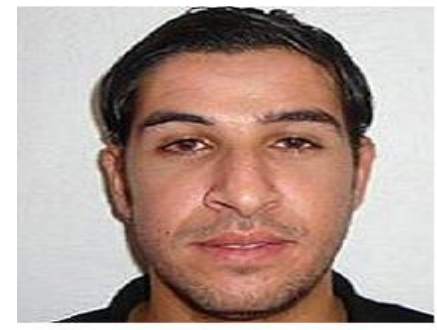

(a) Original Image
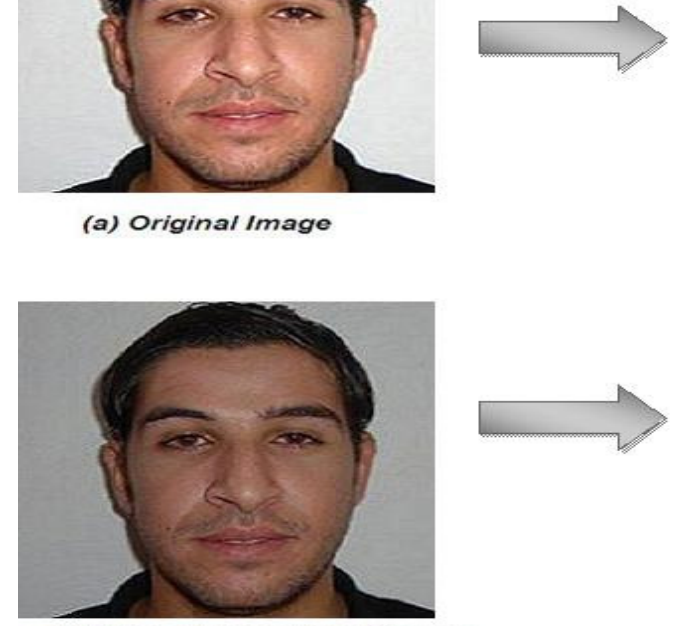

(b) After Illumination Change
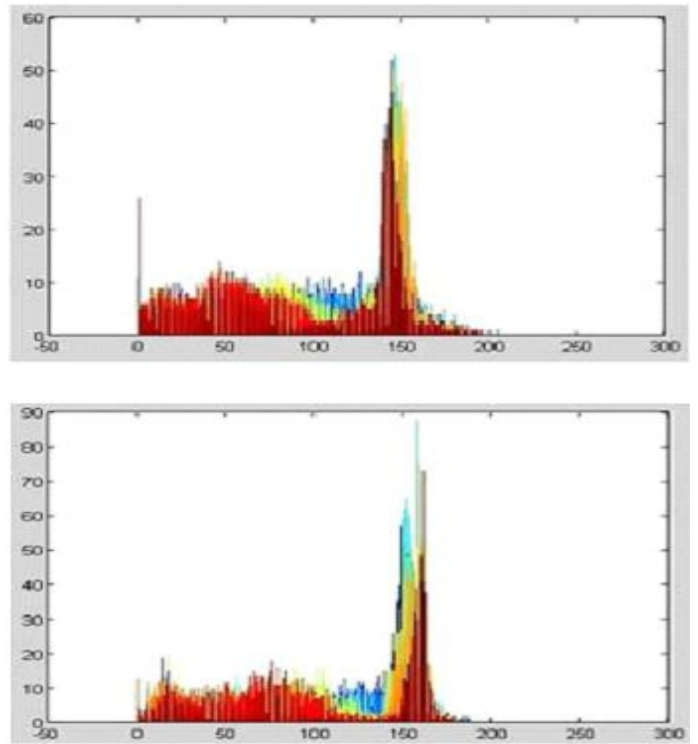

Figure 5. Illumination change (a) Original Image and (b) After Illumination Change.

\section{CONCLUSION}

A kernel machine approach has been presented for learning view-based on for multi-view face detection representations. The main part of the work is the use of KPCA for extracting nonlinear features for each view by learning the nonlinear view-subspace using kernel PCA. This is to construct a mapping view from the input image space, in which the distribution of data points is highly nonlinear and complex. In lower dimensional space the distribution becomes simpler, tighter and therefore more predictable for better modeling of faces. The kernel learning approach leads to an architecture composed of an array of KPCA feature extractors, one for each view. Multi-view face detection is performed by classifying each input image into face or non-face class, by using a two class Kernel Support Vector Classifier (KSVC). Experimental results demonstrate successful face detection approach over a wide range of facial variation in color, illumination conditions, position, scale, orientation, 3D pose, and expression in images from several photo collections. According to the experimental results, the implemented system is high speed and high accuracy makes it appropriate for real time applications such as face tracking and face recognition.

\section{REFERENCES}

[1] James Wayman, Anil Jain, Davide Maltoni and Dario Maio ," Biometric Systems", British Library Cataloguing in Publication Data, (C) Springer-Verlag London Limited 2005.

[2] Christopher A. Waring and Xiuwen Liu," Face Detection Using Spectral Histograms and SVMs", Department of Computer Science, The Florida State University Tallahassee, FL 32306.

[3] Rudy Adipranata , Eddy, Cherry G. Ballangan, and Ronald P. Ongkodjojo ," Fast Method for Multiple Human Face Segmentation in Color Image", International Journal of Advanced Science and Technology Vol. 3, February, 2009.

[4] Min-Quan Jing and Ling-Hwei Chen, " Novel face-detection method under various environments", Optical Engineering 48(6), 067202 (June 2009). 
[5] Lamiaa Mostafa and Sherif Abdelazeem," Face Detection Based on Skin Color Using Neural Networks", GVIP 05 Conference, CICC, Cairo, Egypt, 19-21 December 2005.

[6] Gregory Matthew Wagner, M.S., B.S," Face Authentication with Pose Adjustment Using Support Vector Machines with a Hausdorff-based Image Kernel",Texas Tech University, Gregory Wagner, December 2007.

[7] Peng Wang and Qiang Ji ," Multi-View Face Detection under Complex Scene based on Combined SVMs", Department of Electrical, Computer and System Engineering Rensselaer Polytechnic Institute Troy, NY, 12180.

[8] Ivanna K. Timotius, Iwan Setyawan, and Andreas A. Febrianto ," Face Recognition between Two Person using Kernel Principal Component Analysis and Support Vector Machines", International Journal on Electrical Engineering and Informatics - Volume 2, Number 1, 2010.

[9] Hazim Kemal Ekenel and Bülent Sankur ," Multiresolution face recognition", journal homepage: www.elsevier.com/locate/asoc ,( ElsevierB.V., 2004.

[10] Bernhard Sch olkopf , Alexander Smola , Klaus Robert Muller ," Kernel Principal Component Analysis" , Max-Planck-Institut f. biol. Kybernetik, Spemannstr. 38, 72076 Tubingen, Germany ,2 GMD FIRST, Rudower Chaussee 5, 12489 Berlin, Germany .

[11] Fabio Aiolli and Alessandro Sperduti, " Multiclass Classification with Multi-Prototype Support Vector Machines", Journal of Machine Learning Research 6,2005.

[12] Ignas Kukenys and Brendan McCane,"Support Vector Machines for Human Face Detection",NZCSRSC' Christchurch New Zealand. 2008.

[13] Rik Fransens , Jan De Prins and Luc Van Gool ," SVM-based Nonparametric Discriminant Analysis, An Application to Face Detection",Proceedings of the Ninth IEEE International Conference on Computer Vision (ICCV 2003) 2-Volume Set 0-7695-1950-4/03 \$17.00 (C 2003 IEEE.

[14] Jochen Maydt and Rainer Lienhart ," Face Detection with Support Vector Machines and a Very Large Set of Linear Features", ACM Multimedia '02, December 1-6, 2002.

[15] Phuong-Trinh and Quang-Linh Huynh," Robust Face Detection Under Challenges of Rotation, Pose And Occlusion",Department of Biomedical Engineering, Faculty of Applied Science, Hochiminh University of Technology, Vietnam,2005.

[16] Paola Campadelli and Raffaella Lanzarotti ," Face detection in color images of generic scenes", IEEE International Conference on Computational Intelligence for Homeland Security and Personal Safety Venice, Italy, 21-22 July 2004.

[17] Lamiaa Mostafa and Sherif Abdelazeem ," Face Detection Based on Skin Color Using Neural Networks", GVIP 05 Conference, 19-21 December 2005, CICC, Cairo, Egypt .

[18] Wang Chuan-xu and Li Xue ," Face detection using BP network combined with Gabor wavelet transform" , 978-0-7695-3263-9/08 \$25.00 @ IEEE, 2008.

[19] Jizeng Wang and Hongmei Yang, " Face Detection Based on Template Matching and 2DPCA Algorithm", Lanzhou University of Technology, China, 978-0-7695-3119-9/08 (C) 2008 IEEE.

[20] Min-Quan Jing and Ling-Hwei Chen, " Novel face-detection method under various Environments", National Chiao Tung University,Department of Computer Science, (c) 2009 IEEE.

[21] Yongqiu Tu, Faling Vi, Guohua Chen, Shizhong Jiang and Zhanpeng Huang,"Fast Rotation Invariant Face Detection in Color Image Using Multi-Classifier Combination Method",978-14244-5517-1/10/\$26.00 @ IEEE, 2010.

[22] Payman Moallema, BibiSomayehMousavi, S.AmirhassanMonadjemi, "A novel fuzzy rule base system for pose independent faces detection", journal homepage: www.elsevier.com/locate/asoc, (C) ElsevierB.V., 2010. 


\section{Authors}

${ }^{1}$ Muzhir Shaban Al-Ani has received Ph. D. in Computer \& Communication Engineering Technology, ETSII, Valladolid University, Spain, 1994. Assistant of Dean at Al-Anbar Technical Institute (1985). Head of Electrical Department at Al-Anbar Technical Institute, Iraq (1985-1988), Head of Computer and Software Engineering Department at Al-Mustansyria University, Iraq (1997-2001), Dean of Computer Science (CS) \& Information System (IS) faculty at University of Technology, Iraq (2001-2003). $\mathrm{He}$ joined in 15 September 2003 Electrical and Computer Engineering Department, College of Engineering, Applied Science University, Amman, Jordan, as Associated Professor. He joined in

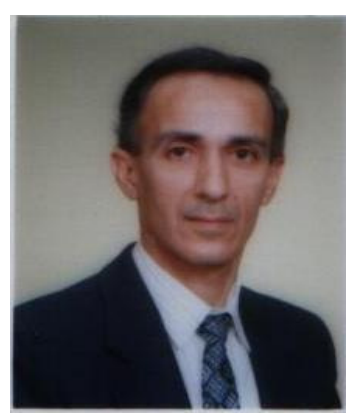
15 September 2005 Management Information System Department, Amman Arab University, Amman, Jordan, as Associated Professor, then he joined computer science department in 15 September 2008 at the same university.

${ }^{2}$ Alaa Sulaiman Nassar Al-Waisy has received B.Sc in Computer Science, Al-Anbar University, Iraq, (2005-2009). M.Sc student (2010- tell now) in Computer Science Department, Al-nabar University. Fields of interest: computer vision, image processing, face recognition, face detection and related fields. Al-Waisy taught many subjects such as operation system, cryptography and encryption.

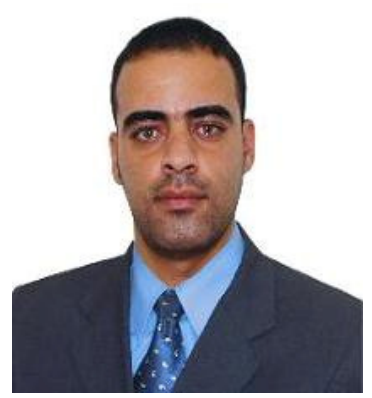

